\title{
Comparación del grosor de tejidos blandos del mentón en individuos con diferentes patrones de crecimiento facial en radiografías cefálicas laterales.
}

Comparison of the soft tissue thickness of the chin in individuals with different patterns of facial growth in lateral cephalic radiographs.

Diana Paola Farez Guichay ${ }^{1, a}$, Miriam Verónica Lima Illescas ${ }^{1, b}$, Magaly Noemí Jiménez
Romero $^{1, c}$, Verónica Ivanova Verdugo Tinitana ${ }^{1, d}$ RESUMEN

Objetivo: Comparar el grosor de tejidos blandos del mentón en individuos con diferentes patrones de crecimiento facial en radiografías cefálicas laterales del cráneo digitales en individuos de 18 a 45 años de edad. Material y Métodos: se realizó un estudio correlacional evaluando 160 radiografías laterales usando una pantalla de 13.3 pulgadas mediante el software AutoCAD 2020 obtenidas de un centro radiológico de la ciudad de Cuenca, en el periodo 2019. Se dividió en cuatro grupos de acuerdo al patrón de crecimiento facial (S-N/G-Gn): Bajo, Medio Bajo, Medio Alto y Alto y el grosor de los tejidos blandos del mentón se midió en sentido sagital a nivel de Pogonion, Gnation y Menton de las estructuras óseas a sus respectivos puntos en los tejidos blandos. Se utilizó la correlación de Rho de Spearman para evaluar las medias del grosor de los tejidos blandos del mentón y patrón facial, para la comparación entre grupos se usó la prueba post-hoc de Tuckey, con un nivel de confiabilidad del 95\% $(p<0,05)$ Resultados: Se encontró diferencias estadísticamente significativas entre las mujeres a nivel de Gnation, mostró un valor $\mathrm{p}=0,041$, el grosor fue mayor en el patrón facial bajo y disminuye según aumenta el patrón esqueletal (Rho Spearman=-0,271) Existió diferencias significativas de acuerdo al dimorfismo sexual a nivel de Menton $(\mathrm{p}=0,049)$ y no existió diferencias significativas de acuerdo a la edad. Conclusión: A medida que las mujeres presentan un patrón facial vertical el espesor de Gnation es más delgado lo que implica, que al modificar verticalmente los tejidos duros de la mandíbula puede afectarse los tejidos blandos.

PALABRAS CLAVE: Mentón, crecimiento, estética, cefalometría. 


\section{ABSTRACT}

Objective: To compare the soft tissue thickness of the chin in individuals with different patterns of facial growth on lateral cephalic radiographs of the digital skull in individuals between 18 and 45 years of age. Material and Methods: a correlational study was carried out evaluating 160 lateral radiographs using a 13.3-inch screen using AutoCAD 2020 software obtained from a radiological center in the city of Cuenca, in the period 2019. It was divided into four groups according to the pattern of facial growth (SN / G-Gn): Low, Medium Low, Medium High and High and the thickness of the soft tissues of the chin was measured sagittally at the Pogonion, Gnation and Menton level of the bone structures at their respective points in soft tissues. The Spearman Rho correlation was used to evaluate the means of the thickness of the soft tissues of the chin and facial pattern, for the comparison between groups the Tuckey post-hoc test was used, with a confidence level of 95\% $(p<0,05)$ Results: Statistically significant differences were found between women at the Gnation level, it showed a value $p=0,041$, the thickness was greater in the low facial pattern and decreases as the skeletal pattern increases (Rho Spearman=-0,271). Significant differences according to sexual dimorphism at the Menton level $(\mathrm{p}=0,049)$ and there were no significant differences according to age. Conclusion: As women present a vertical facial pattern, the Gnation thickness is thinner, which implies that by vertically modifying the hard tissues of the jaw, the soft tissues can be affected.

KEY WORDS: Chin, growth, esthetics, cephalometry.

\section{INTRODUCCIÓN}

La estética facial y la oclusión se encuentran determinados por los tejidos blandos y el crecimiento craneofacial, la relación que existe entre los tejidos blandos de la cara tales como la nariz, los labios y el mentón pueden verse alterados durante el crecimiento, debido a la existencia de una variación individual en diversa magnitud, periodos así como el crecimiento en las distintas partes de la cara; de igual manera, varios estudios evidencian cambios en el perfil de los tejidos blandos después de un tratamiento de ortodoncia $(1,2,3,4)$.

El estudio de la estética facial se ha realizado desde los principios de la ortodoncia, Calvin Case en 1911 estudió los detalles de la cara por medio de máscaras de yeso y mantuvo que las líneas faciales representan un pilar verdadero para un correcto diagnóstico (5). El método auxiliar de diagnóstico en ortodoncia es la cefalometría con la cual se logra planos de referencia ocupando puntos cráneo-cefalométricos, que surgió en 1934 por Hofrath en Alemania y Broadbent en Estados Unidos, generando la posibilidad de emplear una nueva técnica en el estudio de las discrepancias esqueléticas y la maloclusión $(6,7)$.

La apreciación de la estética facial puede variar por la opinión del observador, por el tipo de etnia, raza, edad, dimorfismo sexual, además de otros factores medioambientales que puede influenciar durante el crecimiento craneofacial $(8,9)$. Con respecto al mentón, este es el último hueso craneofacial en culminar su crecimiento, debido a que forma parte de la mandíbula y será alrededor de los 18 a 24 años en hombres mientras que en las mujeres ocurre entre los $20 \mathrm{y}$ 30 años de edad (10).

Los diferentes tejidos blandos que revisten huesos y dientes presentan un grosor variable, los mismos que son mayores en individuos con un patrón de crecimiento hipodivergente, por lo tanto, es necesario entender las diferentes interacciones entre los tejidos duros y blandos $(11,12)$. 
Por su parte, el biotipo facial se define como el conjunto de características morfogenéticas y funcionales que va determinar el patrón de crecimiento y el comportamiento craneofacial de un individuo, mientras que el patrón facial marca la dirección del crecimiento de la cara ya sea en sentido horizontal o vertical. Existe diferentes formas para determinar el biotipo facial tales como: las medidas antropométricas realizadas en el examen clínico y fotográfico, así como a través del trazado cefalométrico. Es importante conocer los cambios que se va a dar durante el crecimiento craneofacial a razón que se puede modificar la mecánica del tratamiento según la necesidad individual $(11,12,13,14)$.

Pacientes adultos han proporcionado información sobre la relación entre los tejidos blandos del mentón y la necesidad de cirugía ortognática en combinación con aparatología fija, ya que la genioplastia está indicada para mejorar los contornos de los tejidos blandos del tercio inferior de la cara debido a la desproporción entre tejidos blandos y duros. Una deficiencia del mentón es reportada en pacientes con un patrón de crecimiento hiperdivergente ya que necesitan una genioplastia de avance junto con un movimiento mandibular $(15,16)$.

Macari y Hanna comparan el grosor de tejidos blandos con varios patrones de divergencia mandibular en la población libanesa (15), Sodawala et al., investigaron en la localidad de la India(1), no existe reportes de estudios en la población ecuatoriana. El objetivo de este estudio fue comparar el grosor de los tejidos blandos del mentón en individuos con diferentes patrones de crecimiento facial en radiografías cefálicas laterales en individuos de 18 a 45 años de edad.

\section{MATERIAL Y MÉTODOS}

Se realizó un estudio observacional y documental, con un nivel relacional. Se usó radiografías cefálicas laterales del cráneo en formato digital de individuos entre 18 y 45 años de edad, tomados en el equipo panorámico dental Newton (Giano) en posición natural de la cabeza, los mismos que pertenecen a un Centro Radiológico de la ciudad de Cuenca, tomadas durante el periodo 2019.

La investigación fue aprobada por el Comité de Ética de la Universidad Católica de Cuenca y el presente estudio no implicó ningún conflicto bioético, debido a que se ejecutó sobre información retrospectiva, por lo que se mantuvo la respectiva confidencialidad de los datos.

Para el tamaño de la muestra se calculó con un nivel de confiabilidad del 95\%, una precisión del 1\% y con una desviación estándar de $3 \mathrm{~mm}$ (1), resultando en 35 radiografías para cada grupo, esta muestra fue ajustada a pérdida de información determinando en una muestra final de 40 individuos para cada tipo facial (bajo, medio bajo, medio alto y alto), con una muestra total de 160 radiografías. El total de telerradiografías que cumplieron los criterios de selección fueron 231 , y se midieron todas las radiografías cefálicas laterales digitales mediante el software AutoCAD 2020 usando una pantalla de 13,3 pulgadas así se determinó el patrón facial, luego se procedió a la selección del tamaño muestral para cada grupo facial a través de un muestreo aleatorio simple.

Las radiografías que cumplieron con los criterios de inclusión fueron telerradiografías de individuos entre 18 y 45 años de edad en el momento de la toma radiográfica, que presenten todas las piezas permanentes a excepción de los terceros molares y los criterios de exclusión fue radiografías cefálicas laterales de individuos con tratamiento de ortodoncia o cirugía ortognática, que presenten anomalías craneofaciales, lesiones o traumatismos en el mentón y contorno no continuo de los tejidos blandos a nivel del mentón.

Una vez seleccionadas las radiografías se llevó a cabo la identificación de puntos óseos y blandos en el mentón, se ejecutó 20 trazados 
cefalométricos durante el día, con el fin de evitar fatiga ocular.

Los datos necesarios fueron número de registro, edad, sexo, patrón de crecimiento facial y grosor del tejido blando del mentón que se midió en 3 niveles, el primero la distancia entre Pogonion óseo (Pog) hasta Pogonion blando (Pog') en proyección horizontal, el segundo fue Gnation óseo (Gn) hasta Gnation de tejido blando (Gn') en dirección oblicua y por último fue Mentón óseo (Me) hasta Mentón de tejido blando (Me') en dirección vertical; mientras que para la determinación del patrón facial se trazó dos planos: el plano mandibular por una línea que une Gonion (Go) y Gnation (Gn) y el plano SN por una línea que une Silla $(\mathrm{S})$ y Nasion $(\mathrm{N})$ a la base craneal (ángulo Go-Gn / S-N), una vez obtenido el ángulo formado entre estos dos planos se dividieron en 4 grupos como: Bajo $\leq$ $27^{\circ}$; Medio Bajo $>27^{\circ}-<32^{\circ}$; Medio Alto $\geq 32^{\circ}$ $<37^{\circ}$; Alto $\geq 37^{\circ}$ (figura 1 ).

Para determinar la confiabilidad intraobservador, un solo investigador repitió las mediciones cefalométricas angular y lineal en
32 radiografías seleccionadas al azar $(20 \%$ de la muestra).

\section{Método estadístico}

El coeficiente de correlación intraclase se utilizó para determinar la variabilidad de las mediciones repetidas. Las diferencias entre la edad y el sexo se evaluaron con la prueba $U$ de Mann-Whitney. Para las comparaciones múltiples entre el patrón de crecimiento facial y el grosor de los tejidos blandos del mentón se utilizó el análisis de la varianza y la prueba post-hoc Tukey, así como también estas pruebas estadísticas se realizó para comparar los parámetros estudiados en hombre y mujeres por separado entre los cuatro grupos, y se usó U de Mann-Whitney para comparar diferencias entre el sexo dentro de cada grupo.

El coeficiente de correlación de Rho de Spearman se utilizó para evaluar las medias del grosor de los tejidos blandos del mentón y patrón facial de crecimiento, la decisión de la relación fue tomada con una consideración del 5\% (p $<0,05)$. El procesamiento de la información, así
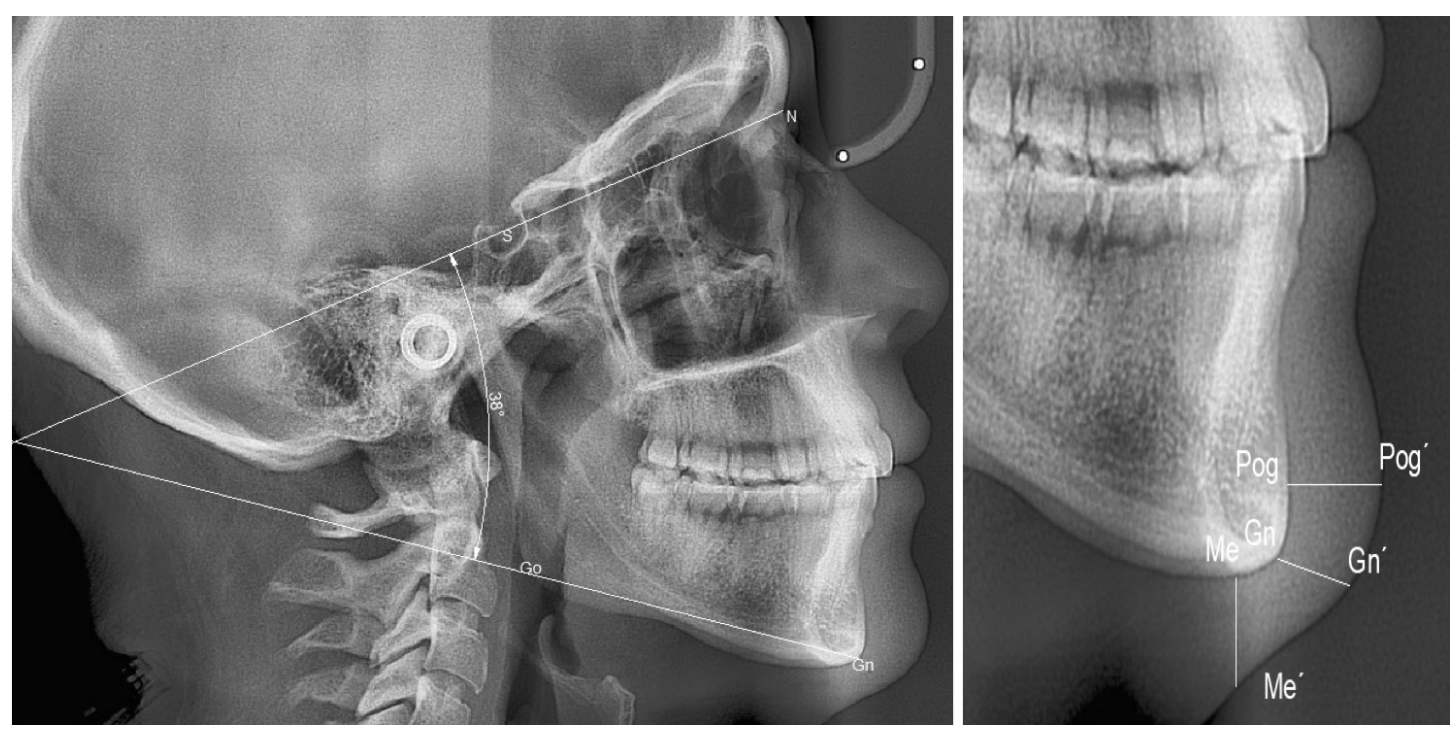

Figura 1. A la izquierda, en la cefalometría lateral se analizó el ángulo Go-Gn / S-N formado por el plano mandibular (Gonion-Gnation) y el plano Silla-Nasion. A la derecha, se evaluó el grosor de los tejidos blandos del mentón desde Pogonion óseo hasta Pogonion de tejido blando en dirección horizontal (Pog-Pog'), la distancia de Gnation óseo a Gnation de tejido blando (Gn-Gn') en dirección oblicua, y la distancia de Mentón óseo hasta Mentón de tejido blando en dirección vertical (Me-Me'). 
como los gráficos se ejecutó en el software SPSS (Statistical Package for the Social Sciences) versión 25.0 y la edición de las tablas se trabajó en Microsoft Excel, 2013.

\section{RESULTADOS}

El presente estudió evaluó 160 radiografías cefálicas laterales de cráneo de individuos entre 18 a 45 años de edad, de las cuales el 45,6\% $(n=73)$ fueron hombres $y$ el $54,4 \% \quad(n=87)$ mujeres.
En la edad no se encontró diferencias estadísticamente significativas para los hombres o las mujeres de acuerdo al patrón de crecimiento facial, e incluso dentro de cada grupo, el detalle de los datos se muestra en la tabla 1.

El promedio del espesor de Pogonion fue de $12,61 \pm 2,01 \mathrm{~mm}$.; a nivel de Gnation fue 10,23 $\pm 2,19 \mathrm{~mm}$; mientras que a nivel de Menton la media fue 8,5 $\pm 2,28 \mathrm{~mm}$. El grosor de los tejidos blandos del mentón al comparar con todos los grupos, muestra que únicamente el grosor

Tabla 1. Medidas descriptivas de acuerdo con la edad

\begin{tabular}{|c|c|c|c|c|c|c|}
\hline Grupos & Total & Bajo & Medio Bajo & Medio Alto & Alto & ANOVA \\
\hline $\mathrm{n}$ & 160 & 40 & 40 & 40 & 40 & $\mathrm{p}$ \\
\hline Edad & $24,83 \pm 6,36$ & $24,90 \pm 6,70$ & $25,18 \pm 6,17$ & $23,6 \pm 6,40$ & $25,65 \pm 6,21$ & 0,52 \\
\hline Hombre & $\begin{array}{c}24,78 \pm 6,44 \\
73\end{array}$ & $\begin{array}{c}24,58 \pm 6,42 \\
26\end{array}$ & $25,63 \pm 5,3919$ & $\begin{array}{c}22,71 \pm 5,78 \\
17\end{array}$ & $\begin{array}{c}27 \pm 8,67 \\
11\end{array}$ & 0,335 \\
\hline $\begin{array}{l}\text { Mujer } \\
\mathrm{n}\end{array}$ & $\begin{array}{c}24,87 \pm 6,33 \\
87\end{array}$ & $25,5 \pm 7,40 \quad 14$ & $24,76 \pm 6,9021$ & $\begin{array}{c}24,26 \pm 6,87 \\
23\end{array}$ & $25,14 \pm 5,0929$ & 0,945 \\
\hline
\end{tabular}

ANOVA $=$ Análisis de la varianza; ${ }^{*} \mathrm{p}<0,05$

Tabla 2. Medias del espesor de los tejidos blandos del mentón según el patrón de crecimiento en los diferentes grupos.

\begin{tabular}{|c|c|c|c|c|c|c|}
\hline \multicolumn{7}{|c|}{ Medias \pm Desviación estándar } \\
\hline Patrón facial & \multicolumn{2}{|c|}{ Bajo } & Medio Bajo & Medio Alto & Alto & ANOVA \\
\hline \multicolumn{6}{|c|}{ Medición de tejidos blandos del mentón (mm.) } & $\mathrm{P}$ \\
\hline Pog-Pog' & $12,54 \pm 2,03$ & & $12,30 \pm 2,40$ & $13,05 \pm 1,71$ & $12,56 \pm 1,83$ & 0,404 \\
\hline Gn-Gn’ & $11,14 \pm 2,44$ & & $10,25 \pm 2,13$ & $10,25 \pm 1,83$ & $9,30 \pm 1,98$ & $0,002 *$ \\
\hline Me-Me' & $8,44 \pm 2,38$ & & $8,89 \pm 2,52$ & $8,84 \pm 2,16$ & $8,20 \pm 2,02$ & 0,473 \\
\hline \multicolumn{7}{|c|}{ Medición Esqueletal $\left(^{\circ}\right)$} \\
\hline S-N/G-Gn & \multicolumn{2}{|l|}{$23,25 \pm 3,24$} & $29,82 \pm 2,40$ & $34,13 \pm 1,15$ & $39,70 \pm 4,04$ & $0,000^{*}$ \\
\hline \multicolumn{7}{|c|}{ Comparación entre grupos } \\
\hline & $\begin{array}{l}\text { Bajo- } \\
\text { Medio Bajo }\end{array}$ & $\begin{array}{l}\text { Bajo- } \\
\text { Medio Alto }\end{array}$ & Bajo- Alto & $\begin{array}{l}\text { Medio Bajo- } \\
\text { Medio Alto }\end{array}$ & $\begin{array}{l}\text { Medio } \\
\text { Bajo- Alto }\end{array}$ & $\begin{array}{c}\text { Medio } \\
\text { Alto-Alto }\end{array}$ \\
\hline \multicolumn{7}{|c|}{ Medición de tejidos blandos del mentón (mm.) } \\
\hline Pog-Pog' & 0,949 & 0,671 & 1 & 0,343 & 0,935 & 0,702 \\
\hline Gn-Gn’ & 0,242 & 0,244 & $0,001 *$ & 1 & 0,186 & 0,185 \\
\hline \multicolumn{7}{|l|}{ Me-Me' } \\
\hline \multicolumn{7}{|c|}{ Medición Esqueletal $\left(^{\circ}\right)$} \\
\hline S-N/G-Gn & $0,000^{*}$ & $0,000 *$ & $0,000^{*}$ & $0,000^{*}$ & $0,000^{*}$ & $0,000 *$ \\
\hline
\end{tabular}


de Gnation fue mayor en un ángulo bajo, y va disminuyendo entre los grupos, siendo menor la distancia en el grupo de ángulo alto, estas diferencias fueron estadísticamente significativa $(\mathrm{p}=0,002)$; al comparar entre los grupos Bajo-
Alto mostró un valor de significancia menor al $5 \%(\mathrm{p}=0,001)$ y su grado de correlación fue baja y negativa $(-0,271)$, los datos se observan en la tabla 2.

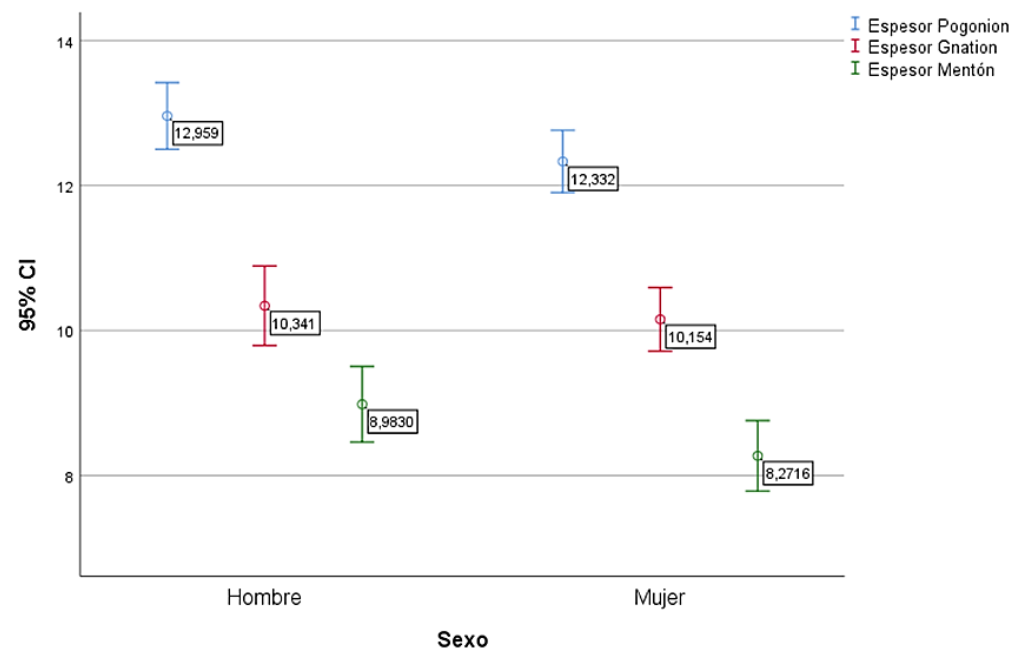

Gráfico 1. Media del grosor del mentón de acuerdo al sexo.

Tabla 3. Medias de los tejidos blandos del mentón en diferentes patrones faciales de acuerdo con el sexo.

\begin{tabular}{|c|c|c|c|c|c|c|}
\hline \multicolumn{5}{|c|}{ Medias \pm Desviación estándar } & \multicolumn{2}{|c|}{ ANOVA } \\
\hline Biotipo Facial & Bajo & Medio Bajo & Medio Alto & Alto & & \\
\hline Hombre (mm) & & & & & \multicolumn{2}{|c|}{$\mathrm{p}$} \\
\hline Pog-Pog' & $12,93 \pm 2,27$ & $12,92 \pm 1,84$ & $13,10 \pm 1,38$ & $12,86 \pm 2,40$ & \multicolumn{2}{|c|}{0,988} \\
\hline Gn-Gn’ & $11,17 \pm 2,82$ & $10,38 \pm 1,94$ & $9,80 \pm 1,60$ & $9,12 \pm 2,25$ & \multicolumn{2}{|c|}{0,066} \\
\hline Me-Me’ & $8,84 \pm 2,70$ & $8,97 \pm 1,86$ & $9,12 \pm 1,42$ & $9,12 \pm 2,82$ & \multicolumn{2}{|c|}{0,976} \\
\hline \multicolumn{7}{|l|}{ Mujer (mm) } \\
\hline Pog-Pog' & $11,83 \pm 1,29$ & $11,74 \pm 2,74$ & $13,01 \pm 1,95$ & $12,45 \pm 1,59$ & \multicolumn{2}{|c|}{0,143} \\
\hline Espesor Gnation & $11,08 \pm 1,61$ & $10,14 \pm 2,34$ & $10,59 \pm 1,95$ & $9,37 \pm 1,90$ & \multicolumn{2}{|c|}{$0,041 *$} \\
\hline Espesor Mentón & $7,71 \pm 1,42$ & $8,82 \pm 3,05$ & $8,64 \pm 2,59$ & $7,85 \pm 1,55$ & \multicolumn{2}{|c|}{0,305} \\
\hline \multicolumn{7}{|c|}{ Comparación entre grupos } \\
\hline & $\begin{array}{l}\text { Bajo-Medio } \\
\text { Bajo }\end{array}$ & $\begin{array}{l}\text { Bajo-Medio } \\
\text { Alto }\end{array}$ & Bajo- Alto & $\begin{array}{l}\text { Medio Bajo- } \\
\text { Medio Alto }\end{array}$ & $\begin{array}{l}\text { Medio } \\
\text { Bajo- Alto }\end{array}$ & $\begin{array}{l}\text { Medio } \\
\text { Alto-Alto }\end{array}$ \\
\hline \multicolumn{7}{|l|}{ Hombre $(\mathrm{mm})$} \\
\hline Pog-Pog' & 1 & 0,992 & 1 & 0,993 & 1 & 0,99 \\
\hline Gn-Gn’ & 0,659 & 0,227 & 0,069 & 0,873 & 0,471 & 0,868 \\
\hline Me-Me' & 0,998 & 0,979 & 0,986 & 0,997 & 0,998 & 1 \\
\hline \multicolumn{7}{|l|}{ Mujer (mm) } \\
\hline Pog-Pog' & 0,999 & 0,3 & 0,77 & 0,154 & 0,596 & 0,743 \\
\hline Gn-Gn’ & 0,523 & 0,886 & $0,048 *$ & 0,877 & 0,523 & 0,543 \\
\hline Me-Me' & 0,493 & 0,625 & 0,998 & 0,994 & 0,448 & 0,6 \\
\hline
\end{tabular}




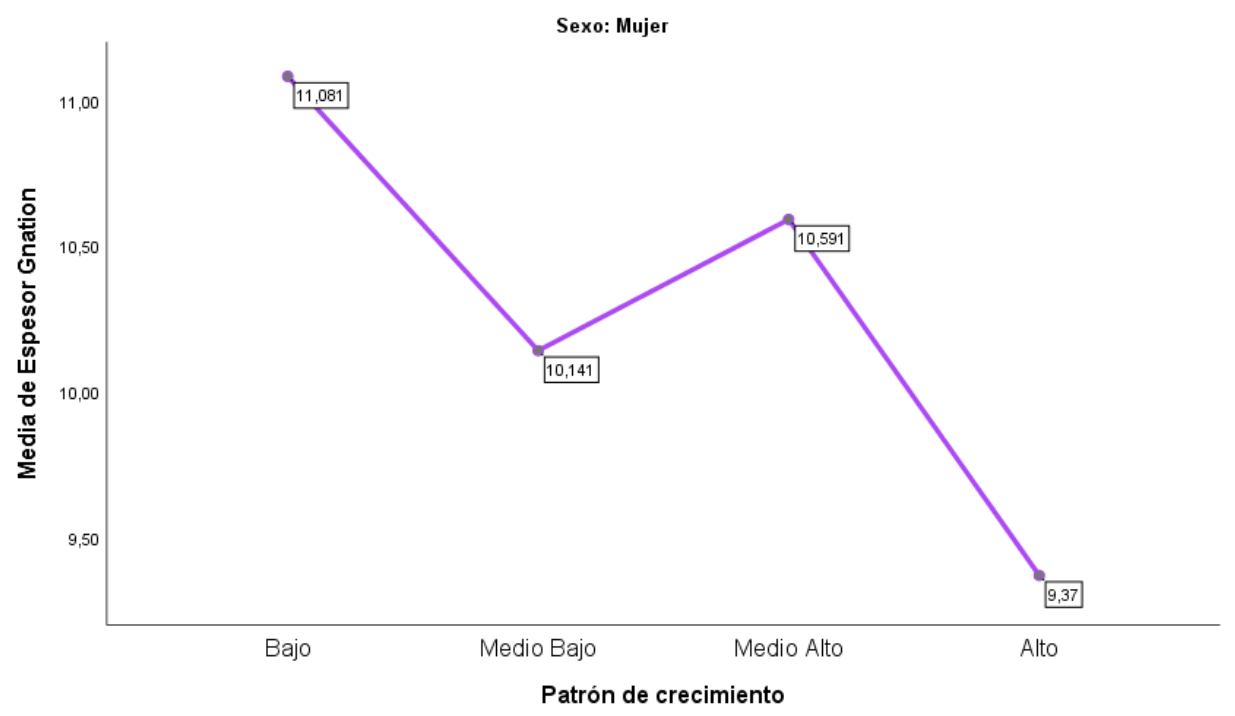

Gráfico 2. Media del espesor de Gnation en relación al patrón de crecimiento en mujeres.

En los hombres el promedio del grosor de Pogonion fue de $12,95 \pm 1,97 \mathrm{~mm}$., a nivel de Gnation fue 10,34 $\pm 2,35 \mathrm{~mm}$., y a nivel de Menton la media fue $8,9 \pm 2,23 \mathrm{~mm}$.; mientras que en las mujeres el promedio del grosor de Pogonion fue de $12,33 \pm 2,01 \mathrm{~mm}$; a nivel de Gnation fue $10,15 \pm 2,05 \mathrm{~mm}$; mientras que a nivel de Menton la media fue 8,27 $\pm 2,28 \mathrm{~mm}$., de acuerdo al sexo se encontró diferencias estadísticamente significativas únicamente a nivel de Menton $(p=0,049)$, se muestra en el gráfico 1 .

Las medias del grosor del mentón entre hombres y mujeres muestran ausencia de diferencias estadísticamente significativa, sin embargo, únicamente nivel del espesor de Gnation mostró diferencias significativas en las mujeres $(\mathrm{p}=0,041)$. Según la prueba post-hoc, al comparar con todos los grupos, las mujeres mostraron diferencia significativa a nivel de Gnation con un valor $\mathrm{p}<0,05$ entre el patrón facial Bajo y Alto; mientras que en los hombres no mostraron diferencias, la información se muestra en la tabla 3 .

En el gráfico 2 se detalla la comparación entre grupos, y se obtuvo que el espesor de Gnation fue mayor en patrón facial bajo y va disminuyendo en comparación al ángulo alto. El coeficiente de correlación intraclase para las mediciones repetidas intraexaminador fue alto $(r=0,982)$ para todas las mediciones.

\section{DISCUSIÓN}

El perfil facial de un individuo va depender del grosor de los tejidos blandos, los mismos que son muy variables por su longitud, grosor y tensión tisular, en especial el espesor del mentón porque además presenta una relación sobre el tejido óseo en la cual se apoya, además influye en la armonía facial que presenta cada individuo (15).

De acuerdo a la edad no se encontró diferencia estadísticamente significativa tanto para hombres como mujeres, concordando con estudios realizados por Sodawala et al., (1), Somaiah et al., (8), y Macari y Hanna (15), en los cuales se analizaron, el patrón de crecimiento con el grosor de tejidos blandos del mentón según la edad del individuo.

El promedio del espesor de los tejidos blandos del mentón fue mayor en el grupo hipodivergente $\mathrm{y}$ fue disminuyendo gradualmente entre los grupos hasta el hiperdivergente similar resultados fueron reportados por Macari y Hanna (15). 
En cuanto al grosor de los tejidos blandos del mentón de acuerdo al patrón de crecimiento se encontró diferencias estadísticamente significativas únicamente a nivel Gnation, éste es más delgado en pacientes con mayor ángulo facial (Rho Spearman $=\quad-0,271)$, a razón de mayor la dimensión vertical de los tejidos esqueléticos menor fue el grosor del tejido blando solo a nivel de Gnation, similar resultado fue reportado por Macari y Hanna(15) sugiriendo que se debe a una mayor extensión de los tejidos blandos a nivel de Gnation por su ubicación en un ángulo a diferencia de Menton cuyo grosor fue menor y menos flexible.

Varios estudios realizados por Sodawala et al., (1), Somaiah et al., (8), y de Gomez et al., (17), al evaluar el grosor del tejido blando del mentón según el sexo en diferentes poblaciones encontraron que el grosor del mentón es mayor en hombres que en mujeres, similar resultado fue encontrado en el presente estudio, a nivel del mentón el promedio en los hombres fue de 8,98 mm., mientras que en las mujeres fue de 8,27 $\mathrm{mm}$.

El promedio de los tejidos blandos del mentón en los diferentes patrones faciales de acuerdo al sexo indicó que el espesor de Gnation mostró diferencia estadísticamente significativa entre las mujeres, ya que el espesor fue mayor en el patrón facial bajo y disminuye según aumenta la dimensión facial vertical, concordando con investigaciones realizadas por Macari y Hanna (15), y Celikoglu et al., (19).

En un estudio realizado por Guerrero en el año 2014, se mostró que la mayoría de individuos mestizos son leptoprosopos donde la dirección de crecimiento vertical es mayor a la horizontal, los individuos con este patrón presentan caras más alargadas y estrechas con un perfil convexo, existiendo comprensión mandibular y maxilar (20). El tercio facial inferior se encuentra aumentado es por eso que la musculatura es débil, haciéndolos más propensos a ser respiradores bucales, presentando un mentón más pequeño y retrognático (11).

Es fundamentalcomprenderque la discrepancia en los resultados con estudios foráneos podría estar ligado a diferencias raciales así como también a factores medioambientales, ya que la mayoría de investigaciones fueron realizados en individuos caucásicos, pero constituye un precedente que hay que tomar en consideración la disposición de los tejidos blandos del mentón especialmente en pacientes hiperdivergentes antes de iniciar una terapia ortodóncica, ya que la consulta del paciente es mejorar la estética facial (18). Dentro de las limitaciones encontradas fue que el trazado se realizó mediante un software de diseño AutoCAD utilizado por arquitectos e ingenieros para crear dibujos precisos en $2 \mathrm{D}$ y 3D, pero al mismo tiempo Mohammed et al., (21), mostraron que la utilización del mismo es confiable en comparación a otros programas de trazados cefalométricos, y ha sido muy utilizado en diferentes estudios $(22,23)$.

\section{CONCLUSIONES}

Con los resultados obtenidos, se concluye que el grosor de los tejidos blandos del mentón, específicamente en Gnation es más delgado en un patrón facial alto en comparación con el bajo en el sexo femenino, lo que implica, que al modificar verticalmente los tejidos duros de la mandíbula puede afectar este tejido blando. De acuerdo al dimorfismo sexual se encontró diferencias significativas a nivel de Menton, pero no se encontraron diferencias significativas en el grosor de los tejidos blandos del mentón según el patrón de crecimiento de acuerdo a la edad.

Conflicto de interés: los autores no tienen conflicto de interés con este informe.

Aprobación de ética: Comité de Ética de la Universidad Católica de Cuenca, Ecuador.

Financiamiento: Ninguno.

Contribuciones de los autores: todos los autores contribuyeron a este manuscrito. 


\section{Correspondencia:}

Diana Paola Farez Guichay

Av. Ordoñez Lasso Cuenca, Ecuador

Correo electrónico: dianafarez28@gmail.com

\section{REFERENCIAS BIBLIOGRÁFICAS}

1. Sodawala J, Akolkar A, Sodawala F, Gandhi S, Hamdani S, Ali S. Comparison of soft tissue chin thickness at different levels of chin in subjects with various growth patterns. Indian J Dent Res. 2020; 31 : 224-228.

2. Kumar A, Tandon P, Singh G, Singh GP. Soft tissue growth changes from 8 to 16 years of age: A crosssectional study. Natl J Maxillofac Surg. 2019; 10 (2): 161-167.

3. Briers N, Briers T, Becker P, Steyn M. Soft tissue thickness values for black and coloured South African children aged 6-13 years. Forensic Science International. 2015; 252: 188-910.

4. Feres M, Hitos S, Sousa H, Matsumoto M. Comparison of soft tissue size between different facial patterns. Dental Press J Orthod. 2010; 15 (4): 84-93.

5. Hoffelder L, Santayana de Lima E, Martinelli F, Bolognese A. Soft-tissue changes during facial growth in skeletal Class II individuals. American Journal of Orthodontics and Dentofacial Orthopedics. 2007; 131 (4): 490-495.

6. Mariel C, Guijarro J, Sánchez W, et al. Estudio transversal comparativo de la relación maxilomandibular de McNamara aplicadas a sujetos mexicanos. Int J Morphol. 2016; 34(2):454-459.

7. Gómez V, Fernández A, Pérez H. Características cefalométricas presentes en la maloclusión clase I en el Departamento de la DEPeI. Rev Odontol Mex 2011; 15(1):14-20.

8. Somaiah S, Khan M, Muddaiah S, Shetty B, Reddy G, Siddegowda R. Comparison of soft tissue chin thickness in adult patients with various mandibular divergence patterns in Kodava population. Int J Orthod Rehabil. 2017; 8 (2): 51-56.

9. Uysal T, Baysal A, Yagci A, Sigler L, McNamara J. Ethnic differences in the soft tissue profiles of Turkish and European-American young adults with normal occlusions and well-balanced faces. The Saudi Dental Journal. 2011; 23(4): 183-190.

10. Cayetano J. Análisis Facial en Ortodoncia. Tesis doctoral. Lima, Perú: Universidad Peruana Cayetano Heredia; 2011. 58 pp.

11. Cerda B, Schulz R, López J, Romo F. Parámetros cefalométricos para determinar biotipo facial en adultos chilenos. Rev Clin Periodoncia Implantol Rehabil Oral. 2019; 12 (1): 8-11.
12. Huanca C, Casas L, Ghersi H. Correlación entre el patrón facial y esquelético de pacientes con deformidad dentofacial clase II. Lima: UPC; 2018.

13. Inda K, Gutiérrez-Rojo J, Gutiérrez-Villaseñor J. Concordancia del biotipo facial con el patrón de crecimiento facial en mujeres y hombres. KIRU. 2019; 16(4): 137-140.

14. Kasai K, Moro T, Kanazawa E, Iwasawa T. Relationship between cranial base and maxillofacial morphology. Eur J Orthod. 1995;17 (5):403-10.

15. Macari A, Hanna A. Comparisons of soft tissue chin thickness in adult patients with various mandibular divergence patterns. The Angle Orthodontist. 2014; 84 (4): 708-714.

16. Maetevorakul S, Viteporn S. Factors influencing soft tissue profile changes following orthodontic treatment in patients with Class II Division 1 malocclusion. Progress in Orthodontics. 2016; 17: 1-13.

17. Gomez Y, Zamora N, Tarazona B, Bellot C, Paredes V. Cross-sectional human study of soft tissue chin (STC) thickness in adult patients in relation to sex, facial pattern and skeletal class. Journal of CranioMaxillofacial Surgery. 2017; 45(8): 1205-1211.

18. Sachan A, Srivastav A, Chaturvedi T. Soft-tissue cephalometric norms in a North Indian ethnic population. J Orthod Sci. 2012;1:92-7.

19. Celikoglu M, Buyuk S, Ekizer A, Sekerci A, Sisman Y. Assessment of the soft tissue thickness at the lower anterior face in adult patients with different skeletal vertical patterns using cone-beam computed tomography. The Angle Orthodontist. 2014; 1: 1-7.

20. Guerrero A. Determinación del biotipo facial y esqueletal de la población ecuatoriana adulta que visita la Clínica Odontológica de la Universidad San Francisco de Quito con oclusión clase I de Angle utilizando análisis cefalométrico de Ricketts, Steiner y BjörkJarabak".Tesis doctoral. Quito, Ecuador: Universidad San Francisco de Quito; 2014. 95 pp.

21. Mohammed N, Ahmed F, Al-Jarad, Zana H. The reliability of AutoCAD program in cephalometric analysis in comparison with pre-programmed cephalometric analysis software. Iraqi Dental Journal. 2012; 34 (1): 35-40.

22. Noor F, Zainab M, Rawof R. Soft Tissue Cephalometric Norms for a Sample of Iraqi Population Group Using Legan and Burstone Analysis. Karbala J Med. 2015; 8 (2):2222-2228.

23. Grewal N, Godhane A. Lateral cephalometry: A simple and economical clinical guide for assessment of nasopharyngeal free airway space in mouth breathers. Contemp Clin Dent. 2010; 1(2): 66-69.

Recibido : 29-03-2021

Aceptado : 13-07-2021 